\title{
Article \\ Bidirectional Electromagnetically Induced Transparency Based on Coupling of Magnetic Dipole Modes in Amorphous Silicon Metasurface
}

\author{
Shuang Liu ${ }^{1}$, Jingxin Dong ${ }^{2}$, Jiangnan $\mathrm{Si}^{1}{ }^{1}$, Weiji Yang ${ }^{1}$, Xuanyi $\mathrm{Yu}^{1}{ }^{1}$, Jialin Zhang ${ }^{1} \subseteq$ and Xiaoxu Deng ${ }^{1, *}$ \\ 1 State Key Laboratory of Advanced Optical Communication Systems and Networks, Key Laboratory for Laser \\ Plasmas (Ministry of Education), School of Physics and Astronomy, Shanghai Jiao Tong University, \\ Shanghai 200240, China; 503313461@sjtu.edu.cn (S.L.); 1359231351370@sjtu.edu.cn (J.S.); \\ yangweiji@sjtu.edu.cn (W.Y.); yxy1593725@sjtu.edu.cn (X.Y.); dorismalfoy@sjtu.edu.cn (J.Z.) \\ 2 School of Electronic Information and Electrical Engineering, Shanghai Jiao Tong University, \\ Shanghai 200240, China; jx_dong@sjtu.edu.cn \\ * Correspondence: xxdeng@sjtu.edu.cn
}

check for updates

Citation: Liu, S.; Dong, J.; Si, J.; Yang, W.; Yu, X.; Zhang, J.; Deng, X. Bidirectional Electromagnetically Induced Transparency Based on Coupling of Magnetic Dipole Modes in Amorphous Silicon Metasurface. Nanomaterials 2021, 11, 1550. https:// doi.org/10.3390/nano11061550

Academic Editor: Andrey

B. Evlyukhin

Received: 20 April 2021

Accepted: 9 June 2021

Published: 11 June 2021

Publisher's Note: MDPI stays neutral with regard to jurisdictional claims in published maps and institutional affiliations.

\begin{abstract}
A bidirectional electromagnetically induced transparency (EIT) arising from coupling of magnetic dipole modes is demonstrated numerically and experimentally based on nanoscale a-Si cuboid-bar metasurface. Analyzed by the finite-difference time-domain (FDTD) Solutions, both the bright and dark magnetic dipole mode is excited in the cuboid, while only the dark magnetic dipole mode is excited in the bar. By breaking the symmetry of the cuboid-bar structure, the destructive interference between bright and dark magnetic dipole modes is induced, resulting in the bidirectional EIT phenomenon. The position and amplitude of simulated EIT peak is adjusted by the vertical spacing and horizontal spacing. The EIT metasurface was fabricated by Electron-Beam Lithography and deep silicon etching technique on the a-Si film deposited by Plasma-Enhanced Chemical Vapor Deposition. Measured by a convergent spectrometer, the fabricated sample achieved a bidirectional EIT peak with transmission up to $65 \%$ and $63 \%$ under forward and backward incidence, respectively. Due to the enhanced magnetic field induced by the magnetic dipole resonance, the fabricated bidirectional EIT metasurface provides a potential way for magnetic sensing and magnetic nonlinearity.
\end{abstract}

Keywords: electromagnetically induced transparency; magnetic dipole modes; a-Si metasurface

\section{Introduction}

Electromagnetically induced transparency (EIT) has attracted enormous interest due to its unique characteristics, including extreme dispersion and enhanced nonlinear effect. So far, an EIT phenomenon has been reported in various systems and structures, among which three-level atomic system [1-5], coupled waveguide [6-13], and metamaterials [14,15] are typical examples. EIT metamaterials require a proper arrangement of geometry structure, of which experimental condition is simple compared with that needed in atomic EIT system. EIT in plasmonic metamaterials has covered a broad range of wavelength from visible to microwave region [16-20] with silver and gold as the chief materials. Graphene-based metamaterials have been proposed to induce tunable EIT effect by adjusting the applied bias on the graphene [21-26], which serve as building blocks for adjustable optical devices. EIT dielectric metamaterials based on Mie-resonance, where the displacement current replaces the conduction current, feature greatly reduced non-radiative loss compared with plasmonic counterparts, providing a promising platform for application in low-loss, slowlight devices [27-29], high-efficiency optical sensors [30,31], polarization convertors [32], and high-modulation optical switches [33,34].

EIT in dielectric metasurfaces has been widely investigated, which originates from the destructive interference between various modes of Mie-resonance, including coupling of 
electric-electric dipole, electric-magnetic dipole, and electric dipole-electric quadrupole. Coupled electric-electric dipole induced transparency has been reported in silicon cross nanostructure metasurface [35], which realizes optical refractive sensing with a figure-ofmerit of 42 and slow-light effect with a group delay of $0.65 \mathrm{ps}$. Coupled electric-magnetic dipole induced transparency has been verified experimentally in silicon ring-bar nanostructure metasurface [36] and disk-bar nanostructure metasurface [37], respectively. The former achieves optical refractive index sensing with a figure-of-merit of 103 and the later generates high-harmonic signal with orders of magnitude higher compared to an unpatterned Si film. Silicon dolmen-like nanostructure metasurface has been employed for realization of coupled electric dipole-electric quadrupole induced transparency [38], of which Q-factor reaches 2680 and group refractive index exceeds 200. However, few works have focused on the EIT behavior which results from the magnetic-magnetic dipole coupling in dielectric metasurfaces.

In this paper, a bidirectional EIT induced by coupling between magnetic dipoles is analyzed numerically and experimentally in nanoscale a-Si cuboid-bar metasurface. Based on discrete dipole approximation (DDA) theory, the magnetic dipoles in both cuboid and bar nanostructure, treated as bright and dark mode, are calculated from the simulated displacement currents, which interfere destructively to generate EIT phenomenon. The impact of spacing between cuboid and bar on the EIT peak is investigated by numerical simulations, and the results indicate that the EIT peak is tuned by the coupling separations and structural asymmetry, which is the spacing between cuboid and bar in different direction, respectively, arising from the shift of the dark mode and change of coupling effect. The bidirectional EIT metasurface was fabricated by Electron-Beam Lithography (EBL) and deep silicon etching technique. The transmission spectra of the fabricated EIT metasurface are measured by a convergent spectrometer, which exhibit a bidirectional EIT peak with transmission up to $65 \%$ and $63 \%$ under forward and backward incidence, respectively. Due to the enhanced magnetic field induced by the magnetic dipole Mie resonance inside the cuboid and bar nanostructure and near-zero nonradiative loss of a-Si, the proposed bidirectional EIT metasurface which realize EIT phenomenon under bidirectional incidence, has potential applications in the field of magnetic sensing, magnetic nonlinearity and bidirectional devices.

\section{Materials and Methods}

The schematic of the near-infrared bidirectional EIT metasurface is shown in Figure 1a, which is an a-Si cuboid-bar nanostructure periodic array on the $\mathrm{SiO}_{2}$ substrate. The unit cell of the periodic array, which comprises a cuboid resonator and a bar resonator, is shown in Figure $1 \mathrm{~b}$. The side length of the cuboid is $\mathrm{a}=150 \mathrm{~nm}$. The length and width of the bar is $\mathrm{b}=230 \mathrm{~nm}$ and $\mathrm{c}=70 \mathrm{~nm}$, respectively. The height of the two resonators is $\mathrm{h}=270 \mathrm{~nm}$. The center distance between two resonators in $\mathrm{x}$ and $\mathrm{y}$ direction (along the short and long axis of the bar) is $g$ and $f$, respectively. The period of unit cell in $x$ and $y$ direction is $P=500 \mathrm{~nm}$. The refractive index of $\mathrm{SiO}_{2}$ is 1.528 . 


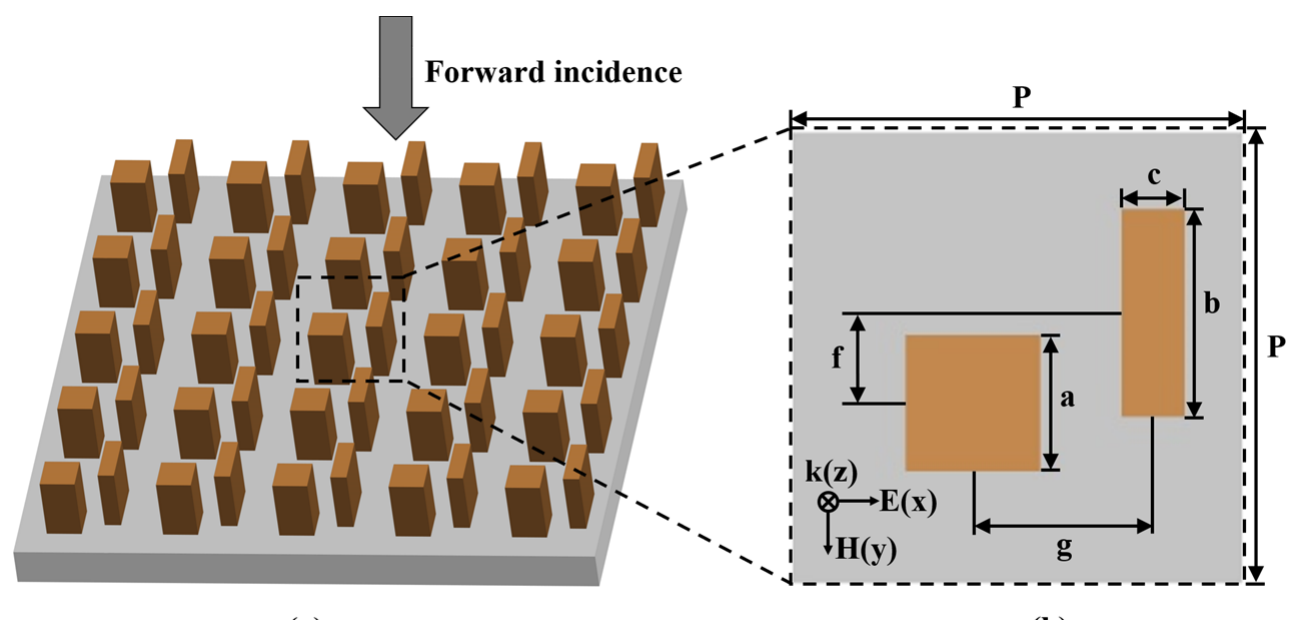

(a)

(b)

Figure 1. (a) Schematic of the near-infrared bidirectional EIT metasurface based on a-Si cuboid-bar nanostructure periodic arrays on the $\mathrm{SiO}_{2}$ substrate. The black dash box shows a unit cell. (b) Top view of the unit cell.

Transmission spectra and displacement currents of the metasurfaces are simulated by finite-difference time-domain (FDTD) Solutions. In the simulations, a semi-infinite substrate is added below the metasurfaces, and a monitor in the substrate is set $500 \mathrm{~nm}$ below the metasurfaces to calculate the transmission from the ratio of the power flowing into the monitor to the power of light source. $x$ component and $y$ component of magnetic dipole moments inside the cuboid and the bar of the bidirectional EIT metasurface are calculated based on discrete dipole approximation (DDA) theory [39,40], which is given by:

$$
\begin{gathered}
m_{1}=m_{y c u b o i d}=\frac{1}{2} \int_{\text {cuboid }} z J_{x}-x J_{z} d^{3} r \\
m_{2}=m_{x b a r}=\frac{1}{2} \int_{\text {bar }} y J_{z}-z J_{y} d^{3} r \\
m_{3}=m_{x c u b o i d}=\frac{1}{2} \int_{\text {cuboid }} y J_{z}-z J_{y} d^{3} r
\end{gathered}
$$

where $J_{x}, J_{y}$ and $J_{z}$ is the $x, y$ and $z$ component of displacement current, respectively. The discrete dipole approximation decomposes the cuboid and bar nanostructures into multiple point dipoles, each of which generates a displacement current which is used to calculate the magnetic dipole moments.

The correlation coefficient is introduced to evaluate the matching between the numerical and experimental EIT peaks, which is given by:

$$
r\left(T_{\text {sim }}, T_{\text {exp }}\right)=\frac{\sum\left(T_{\text {sim }}-\bar{T}_{\text {sim }}\right)\left(T_{\text {exp }}-\bar{T}_{\text {exp }}\right)}{\sqrt{\sum\left(T_{\text {sim }}-\bar{T}_{\text {sim }}\right)^{2}\left(T_{\text {exp }}-\bar{T}_{\text {exp }}\right)^{2}}},
$$

where $T_{\text {sim }}, T_{\exp }$ is the simulated and experimental transmissions of the EIT peak, respectively, and $\bar{T}_{\text {sim }}, \bar{T}_{\text {exp }}$ is the average simulated and experimental transmission of the EIT peak.

An a-Si film is grown on the $\mathrm{SiO}_{2}$ substrate by Plasma-Enhanced Chemical Vapor Deposition (PECVD). The thickness of the $\mathrm{SiO}_{2}$ substrate is $1 \mathrm{~mm}$. The cuboid-bar nanostructure EIT metasurface is fabricated by electron-beam lithography and deep silicon etching technique. The transmission spectra of the fabricated cuboid-bar nanostructure EIT metasurface are measured by a convergent spectrometer. As shown by Figure 2, a polarizer is added in the convergent spectrometer to ensure that the incident light is polarized along the short axis of the bar in the bidirectional EIT metasurface. 


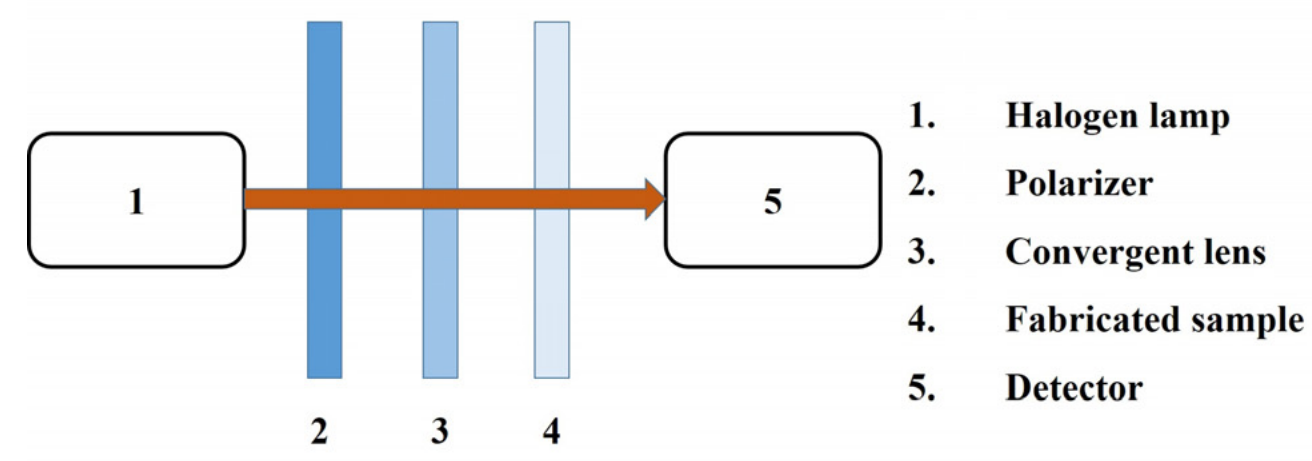

Figure 2. The schematic presentation of the convergent spectrometer with a polarizer.

\section{Results and Discussion}

\subsection{Numerical and Simulated Results}

Transmission spectra for sole-cuboid nanostructure metasurface, sole-bar nanostructure metasurface and bidirectional EIT metasurface are simulated by FDTD Solutions. The simulated transmission spectrum of the sole-cuboid nanostructure metasurface with the incident electric field E along $x$ axis exhibits a dip at 803 and at $722 \mathrm{~nm}$ under backward incidence, as shown by black curve in Figure 3b. At the transmission dip $803 \mathrm{~nm}$, the enhanced circular electric field in the cuboid simulated by FDTD at the $\mathrm{x}-\mathrm{z}$ plane corresponds to magnetic dipole resonance oriented in y direction, which forms the bright mode shown in Figure 3c. At the transmission dip $722 \mathrm{~nm}$, the enhanced linear electric field in the cuboid simulated by FDTD at the $x-y$ plane corresponds to electric dipole resonance oriented in $x$ direction shown in Figure 3d. Under forward incidence, the dip at $803 \mathrm{~nm}$ remains unchangeable while the dip at $722 \mathrm{~nm}$ reduces significantly in the simulated transmission spectrum of sole-cuboid nanostructure metasurface shown by the black curve in Figure 3a, indicating that the magnetic dipole mode is bidirectional but the electric dipole mode is not. The scattering electric field induced by magnetic dipole is related to the permeability, which remains unchangeable under bidirectional incidence as the permeability is constant to 1 for both a-silicon metasurfaces and substrate. While, the scattering electric field induced by electric dipole is related to the permittivity, which changes with the direction of incidence as the permittivity distribution is different under forward incidence (incident from air side) and backward incidence (incident from substrate side). Due to the $C_{4}$ symmetry of the cuboid, magnetic dipole resonance oriented in $\mathrm{x}$ direction is also excited by the $\mathrm{E}$ along $y$ axis at $803 \mathrm{~nm}$ and cannot be excited by the x-polarized light directly, which serves as the dark mode under $x$-polarized incidence. In the simulated transmission spectrum of the sole-bar nanostructure metasurface with the incident electric field $\mathrm{E}$ along $\mathrm{y}$ axis under bidirectional incidence, a pronounced dip, as shown by red curve in Figure 3a,b, occurs at $806 \mathrm{~nm}$, around which circular electric field at the y-z plane of the bar is simulated in Figure $3 \mathrm{e}$, so a bidirectional magnetic dipole resonance in $\mathrm{x}$ direction forms and serves as the dark mode under x-polarized incidence. A transparency window centered at $809 \mathrm{~nm}$ is observed in the simulated transmission spectrum of the bidirectional EIT metasurface with the incident electric field $\mathrm{E}$ along $\mathrm{x}$ axis under bidirectional incidence, as shown by blue curve in Figure 3a,b. The simulated electric field distribution at the EIT peak inside the bar resonator and the cuboid resonator at the y-z plane both exhibit an enhanced loop, as shown in Figure 3f,g, respectively, whereas the circular electric field inside the cuboid resonator at the x-z plane vanishes, as shown in Figure 3h. Hence, the dark modes not only in the bar resonator but also in the cuboid resonator are excited simultaneously by the bright mode other than external electric field. When the structural asymmetry is introduced to the metasurface, the bright bidirectional magnetic dipole (MD) mode and dark bidirectional MD mode, arranged in close proximity in the special and frequency domain, interfere with 
each other destructively, suppressing the bright mode and consequently leading to the bidirectional EIT phenomenon.
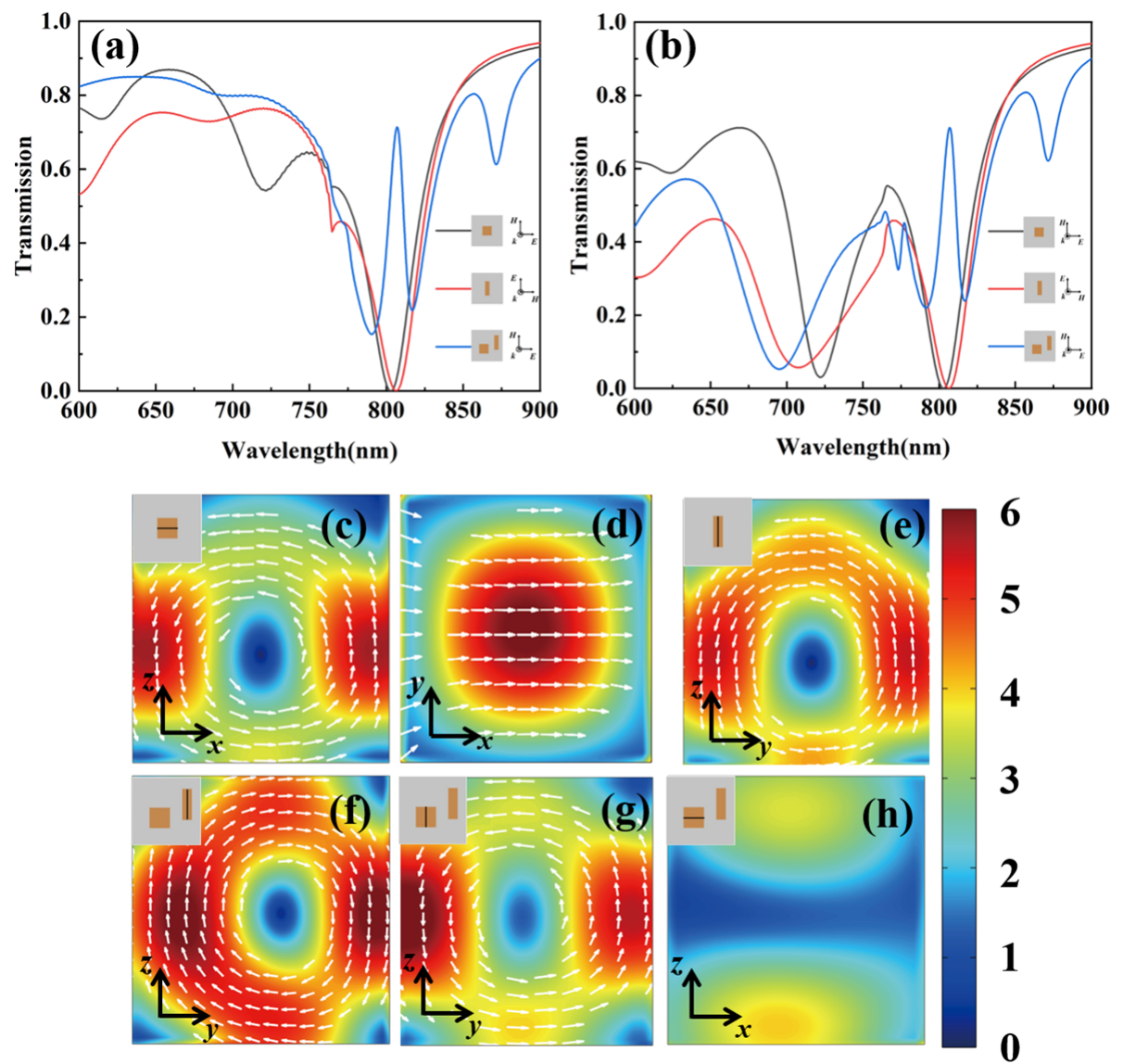

Figure 3. Simulated transmission spectrum for sole-cuboid nanostructure metasurface, sole-bar nanostructure metasurface and bidirectional EIT metasurface with $\mathrm{f}=100 \mathrm{~nm}$ and $\mathrm{g}=200 \mathrm{~nm}$ under forward incidence (a) and backward incidence (b). Distribution of electric field in x-z plane inside the cuboid of the sole-cuboid nanostructure metasurface at $803 \mathrm{~nm}$ (c), in x-y plane inside the cuboid of the sole-cuboid nanostructure metasurface at $722 \mathrm{~nm}(\mathbf{d})$, in $y-z$ plane inside the bar of the sole-bar nanostructure metasurface at $806 \mathrm{~nm}$ (e). Distribution of electric field in $\mathrm{y}-\mathrm{z}$ plane inside the bar (f), in $y-z$ plane inside the cuboid (g), and in $x-z$ plane inside the cuboid (h) of the near-infrared bidirectional EIT metasurface at the EIT peak.

The magnetic dipole moments in the bidirectional EIT metasurface are calculated from the displacement currents simulated by FDTD. The magnetic dipole moment $m_{1}$ in cuboid ( $x$ component of the magnetic dipole), which relates to bright mode, reaches minimum at $809 \mathrm{~nm}$, shown by red curve in Figure 4, whereas the magnetic dipole moments $m_{2}$ in bar and $m_{3}$ in cuboid ( $y$ component of the magnetic dipole), which relate to dark mode, both exhibit a peak at $810 \mathrm{~nm}$, as shown by black curve and blue curve in Figure 4, respectively. The maximum of the magnetic dipole moments $m_{2}$ and $m_{3}$ corresponds to the minimum of the magnetic dipole moment $m_{1}$, that is, the enhancement of the dark mode is accompanied by the decrease of the bright mode. The electromagnetic energy is transferred from the bright mode $m_{1}$ into the dark modes $m_{2}$ and $m_{3}$ due to the near-field coupling, resulting in the dip of $m_{1}$ and the peak of $m_{2}$ and $m_{3}$. The destructive interference between bright and dark modes results in the EIT phenomenon in cuboid-bar metasurface. 


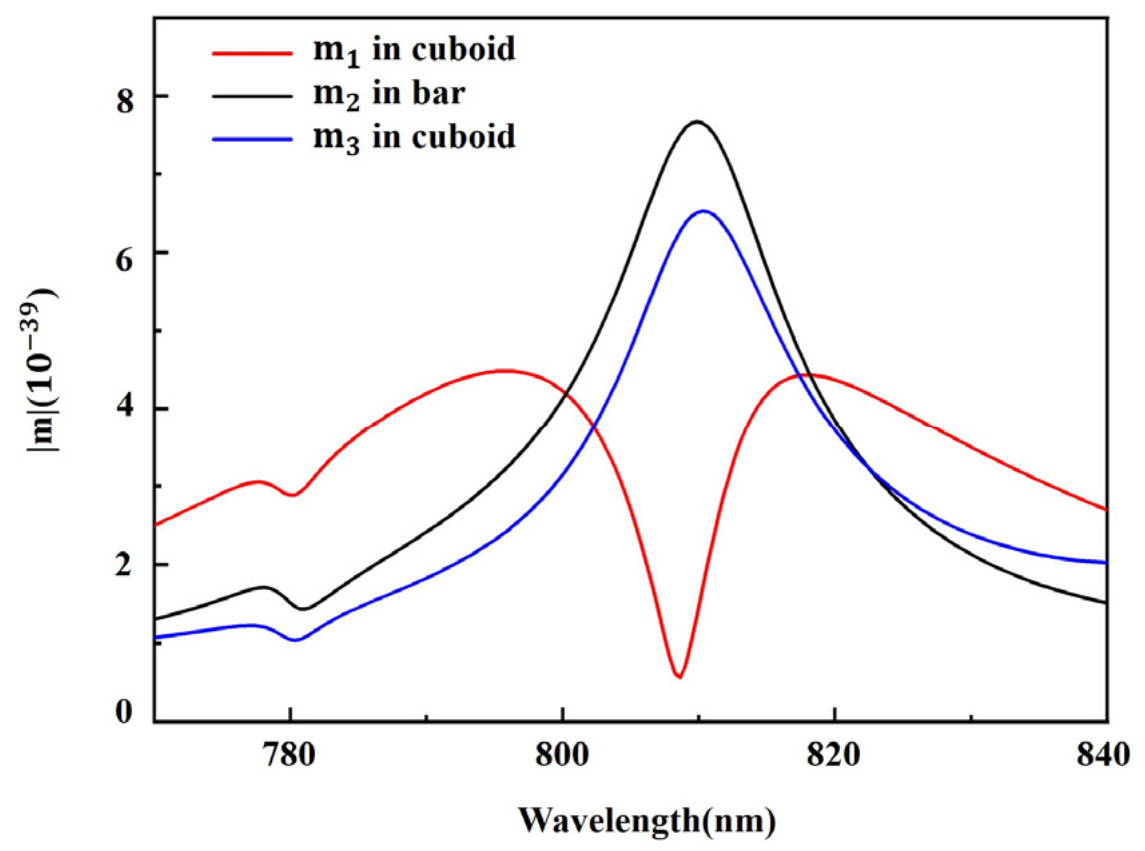

Figure 4. Calculated magnetic dipole moment inside the cuboid and the bar of the bidirectional EIT metasurface.

The transmission spectra of the cuboid-bar nanostructure EIT metasurface are simulated by FDTD Solutions with vertical spacing $f$ and horizontal spacing $g$. The simulated transmission spectra of EIT metasurface with different vertical spacing $f$ are shown in Figure $5 \mathrm{a}$, where the EIT peak increases and red-shifts with vertical spacing $\mathrm{f}$ varying from 0 to $100 \mathrm{~nm}$. When $\mathrm{f}$ equals to 0 , the EIT phenomenon vanishes and only the MD resonance dip remains in the simulated transmission spectrum. Increasing structural asymmetry $\mathrm{f}$ will enhance the coupling effect between the cuboid and bar, and change the spatial location of the center of the circular displacement currents inside the bar, shown by inset in Figure $5 \mathrm{a}$, resulting in red-shift of the dark modes and consequently shift and enhancement of the EIT peak. The simulated transmission spectra of EIT metasurface with horizontal spacing g are shown in Figure 5b. When the horizontal spacing g increases from 190 to $230 \mathrm{~nm}$, the EIT peak increases and broadens with the spectral position fixed at $809 \mathrm{~nm}$ as a result of enhancement of coupling among the magnetic dipoles in cuboid and bar.
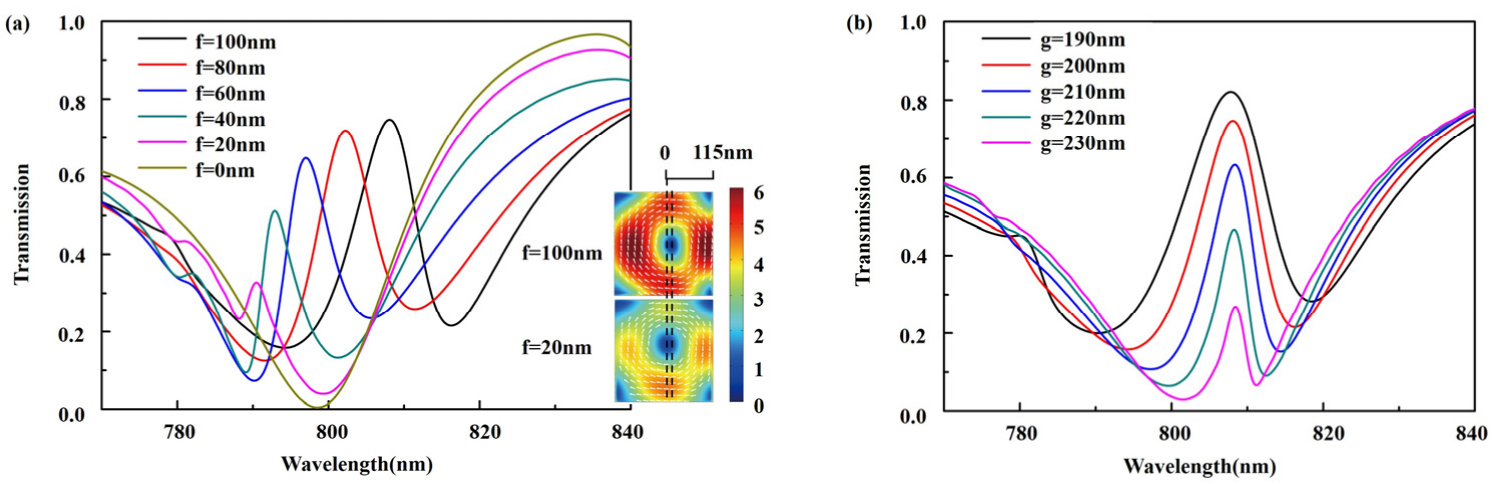

Figure 5. Simulated transmission spectrum of the periodic cuboid-bar nanostructure EIT metasurface with (a) different vertical spacing, (b) different horizontal spacing g between the cuboid and the bar. Inset shows the distribution of electric field with displacement currents in $y$ - $z$ plane inside the bar when $f=20 \mathrm{~nm}$ and $f=100 \mathrm{~nm}$ at the EIT peak. 


\subsection{Experimental Results}

Scanning electron microscopy (SEM) image of the fabricated sample is shown in Figure 6a. The size of the cuboid and bar is close to $150 \mathrm{~nm} \times 150 \mathrm{~nm}, 230 \mathrm{~nm} \times 70 \mathrm{~nm}$, respectively. Period of the cuboid-bar array nanostructure is $\mathrm{P}_{\mathrm{x}} \approx 500 \mathrm{~nm}, \mathrm{P}_{\mathrm{y}} \approx 500 \mathrm{~nm}$. The total area of the fabricated sample is $300 \mu \mathrm{m} \times 300 \mu \mathrm{m}$. The measured transmission spectra of the fabricated EIT metasurface exhibit a bidirectional EIT peak with a maximum of $65 \%$ and a maximum of $63 \%$ at $806 \mathrm{~nm}$ under forward and backward incidence, respectively, shown by black curve in Figure $6 \mathrm{~b}, \mathrm{c}$. The experimental spectra are in the same trend with the simulated results shown by red curve in Figure $6 \mathrm{~b}, \mathrm{c}$, despite that the EIT peak in the measured spectra is reduced and broadened, which arises from the imperfection of the fabricated EIT metasurfaces and the formation of a low refractive index oxide layer. The correlation coefficient between the experimental and simulated EIT peak reaches 0.73 and 0.78 under forward and backward incidence, respectively, which demonstrates the good matching between the measured and simulated EIT peak.

(a)
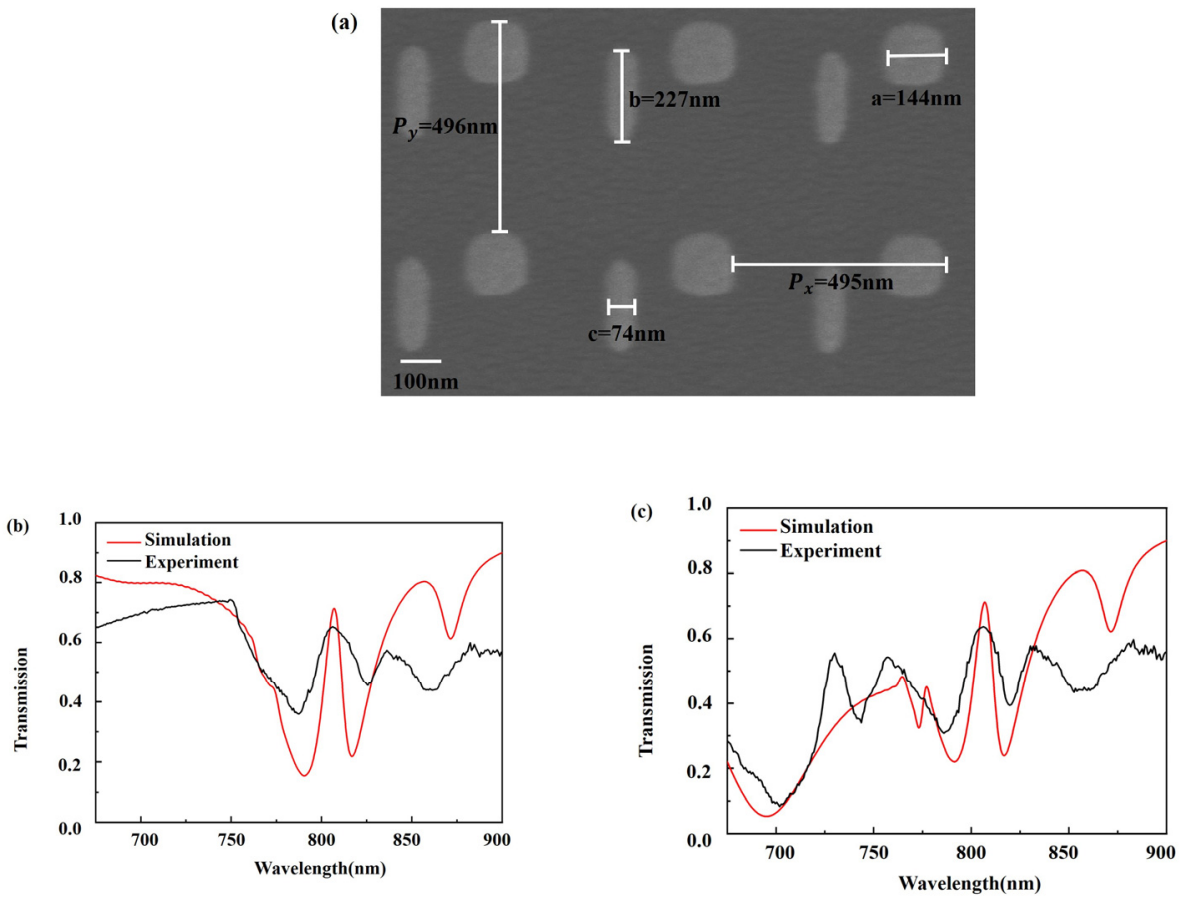

Figure 6. (a) SEM image of the fabricated bidirectional EIT metasurface. The measured and simulated transmission spectrum of the bidirectional EIT metasurface (b) under forward incidence, (c) under backward incidence.

\section{Conclusions}

In conclusion, a nanoscale a-Silicon cuboid-bar metasurface is investigated numerically and demonstrated experimentally to achieve bidirectional EIT response induced by the near field coupling between magnetic dipole modes. The cuboid supports both bright and dark magnetic dipole mode, while the bar only supports dark magnetic dipole mode. When the structural symmetry of metasurface is broken, EIT stems from the destructive interference between bright and dark magnetic dipole modes. Simulated by FDTD Solutions, the EIT peak shifts and fluctuates according to the vertical spacing (structural asymmetry) and horizontal spacing between cuboid and bar, which is caused by the relocation of the center of the displacement currents and change of coupling effect. A convergent spectrometer is utilized to measure the transmission spectra of the EIT metasurface which is fabricated by Electron-Beam Lithography (EBL) and deep silicon etching technique. The EIT peak in experiments arrives $65 \%$ and $63 \%$ for light incident from front and back side, respectively, which exhibits the same trend as the simulations. Due to the enhanced magnetic field 
induced by the magnetic dipole Mie resonance and near-zero nonradiative loss of a-Si, the proposed bidirectional EIT phenomenon is prospective in the field of magnetic sensing and magnetic nonlinearity.

Author Contributions: Software, S.L., J.Z. and X.Y.; validation, W.Y. and J.D.; formal analysis, J.S., S.L., and W.Y.; data curation, S.L.; writing—original draft preparation, S.L.; writing—review and editing, X.D.; funding acquisition, X.D. All authors have read and agreed to the published version of the manuscript.

Funding: This research was funded by National Natural Science Foundation of China, grant numbers 61927822 .

Institutional Review Board Statement: Not applicable.

Informed Consent Statement: Not applicable.

Data Availability Statement: The data presented in this study are available on request from the corresponding author.

Acknowledgments: The Center of Advanced Electronic Materials and Devices (AEMD) of Shanghai Jiao Tong University support us to fabricate the nanoscale amorphous silicon metasurfaces.

Conflicts of Interest: The authors declare no conflict of interest.

\section{References}

1. Hau, L.V.; Harris, S.E.; Dutton, Z.; Behroozi, C.H. Light speed reduction to 17 metres per second in an ultracold atomic gas. Nature 1999, 397, 594-598. [CrossRef]

2. Fleischhauer, M.; Imamoglu, A.; Marangos, J.P. Electromagnetically induced transparency: Optics in coherent media. Rev. Mod. Phys. 2005, 77, 633-673. [CrossRef]

3. Jia, F.; Zhang, J.; Zhang, L.; Wang, F.; Mei, J.; Yu, Y.; Zhong, Z.; Xie, F. Frequency stabilization method for transition to a Rydberg state using Zeeman modulation. Appl. Opt. 2020, 59, 2108-2113. [CrossRef] [PubMed]

4. Wen, Y.; Zhou, P.; Xu, Z.; Yuan, L.; Wang, M.; Wang, S.; Chen, L.; Wang, H.; Shengzhi, W. Cavity-enhanced and long-lived optical memories for two orthogonal polarizations in cold atoms. Opt. Express 2020, 28, 360-368. [CrossRef]

5. Solomons, Y.; Banerjee, C.; Smartsev, S.; Friedman, J.; Eger, D.; Firstenberg, O.; Davidson, N. Transverse drag of slow light in moving atomic vapor. Opt. Lett. 2020, 45, 3431. [CrossRef] [PubMed]

6. Wang, T.; Hu, Y.-Q.; Du, C.-G.; Long, G.-L. Multiple EIT and EIA in optical microresonators. Opt. Express 2019, $27,7344-7353$. [CrossRef] [PubMed]

7. Smith, D.D.; Chang, H.; Fuller, K.A.; Rosenberger, A.; Boyd, R.W. Coupled-resonator-induced transparency. Phys. Rev. A 2004, 69, 063804. [CrossRef]

8. Xu, Q.; Sandhu, S.; Povinelli, M.L.; Shakya, J.; Fan, S.; Lipson, M. Experimental Realization of an On-Chip All-Optical Analogue to Electromagnetically Induced Transparency. Phys. Rev. Lett. 2006, 96, 123901. [CrossRef] [PubMed]

9. Jiang, F.; Deng, C.-S.; Lin, Q.; Wang, L.-L. Simulation study on active control of electromagnetically induced transparency analogue in coupled photonic crystal nanobeam cavity-waveguide systems integrated with graphene. Opt. Express 2019, 27, 32122-32134. [CrossRef]

10. Han, Y.; Yang, J.; He, X.; Huang, J.; Zhang, J.; Chen, D.; Zhang, Z. High quality factor electromagnetically induced transparencylike effect in coupled guided-mode resonant systems. Opt. Express 2019, 27, 7712-7718. [CrossRef]

11. Tang, H.; Zhou, L.; Xie, J.; Lu, L.; Chen, J. Electromagnetically Induced Transparency in a Silicon Self-Coupled Optical Waveguide. J. Light. Technol. 2018, 36, 2188-2195. [CrossRef]

12. Lu, H.; Gan, X.; Mao, D.; Zhao, J. Graphene-supported manipulation of surface plasmon polaritons in metallic nanowaveguides. Photon. Res. 2017, 5, 162-167. [CrossRef]

13. Keleshtery, M.H.; Kaatuzian, H.; Mir, A.; Zandi, A. Method proposing a slow light ring resonator structure coupled with a metal-dielectric-metal waveguide system based on plasmonic induced transparency. Appl. Opt. 2017, 56, 4496-4504. [CrossRef]

14. Zhang, S.; Genov, D.A.; Wang, Y.; Liu, M.; Zhang, X. Plasmon-Induced Transparency in Metamaterials. Phys. Rev. Lett. 2008, 101, 047401. [CrossRef] [PubMed]

15. Pitchappa, P.; Manjappa, M.; Ho, C.P.; Singh, R.; Singh, N.; Lee, C. Active Control of Electromagnetically Induced Transparency Analog in Terahertz MEMS Metamaterial. Adv. Opt. Mater. 2016, 4, 541-547. [CrossRef]

16. Dong, Z.-G.; Liu, H.; Cao, J.-X.; Li, T.; Wang, S.-M.; Zhu, S.-N.; Zhang, X. Enhanced sensing performance by the plasmonic analog of electromagnetically induced transparency in active metamaterials. Appl. Phys. Lett. 2010, 97, 114101. [CrossRef]

17. Wan, M.; Yuan, S.; Dai, K.; Song, Y.; Zhou, F. Electromagnetically induced transparency in a planar complementary metamaterial and its sensing performance. Optik 2015, 126, 541-544. [CrossRef]

18. Wan, M.; Song, Y.; Zhang, L.; Zhou, F. Broadband plasmon-induced transparency in terahertz metamaterials via constructive interference of electric and magnetic couplings. Opt. Express 2015, 23, 27361-27368. [CrossRef] [PubMed] 
19. Zhao, Z.; Gu, Z.; Ako, R.T.; Zhao, H.; Sriram, S. Coherently controllable terahertz plasmon-induced transparency using a coupled Fano-Lorentzian metasurface. Opt. Express 2020, 28, 15573-15586. [CrossRef]

20. Duan, X.; Chen, S.; Cheng, H.; Li, Z.; Tian, J. Dynamically tunable plasmonically induced transparency by planar hybrid metamaterial. Opt. Lett. 2013, 38, 483-485. [CrossRef]

21. Xiao, B.; Tong, S.; Fyffe, A.; Shi, Z. Tunable electromagnetically induced transparency based on graphene metamaterials. Opt. Express 2020, 28, 4048-4057. [CrossRef] [PubMed]

22. Sun, C.; Si, J.; Dong, Z.; Deng, X. Tunable multispectral plasmon induced transparency based on graphene metamaterials. Opt. Express 2016, 24, 11466-11474. [CrossRef] [PubMed]

23. Dong, Z.; Sun, C.; Si, J.; Deng, X. Tunable polarization-independent plasmonically induced transparency based on metal-graphene metasurface. Opt. Express 2017, 25, 12251-12259. [CrossRef] [PubMed]

24. Sun, C.; Dong, Z.; Si, J.; Deng, X. Independently tunable dual-band plasmonically induced transparency based on hybrid metal-graphene metamaterials at mid-infrared frequencies. Opt. Express 2017, 25, 1242. [CrossRef]

25. Lao, C.; Liang, Y.; Wang, X.; Fan, H.; Wang, F.; Meng, H.; Guo, J.; Liu, H.; Wei, Z. Dynamically Tunable Resonant Strength in Electromagnetically Induced Transparency (EIT) Analogue by Hybrid Metal-Graphene Metamaterials. Nanomaterials 2019,9 , 171. [CrossRef]

26. Sun, G.; Peng, S.; Zhang, X.; Zhu, Y. Switchable Electromagnetically Induced Transparency with Toroidal Mode in a GrapheneLoaded All-Dielectric Metasurface. Nanomaterials 2020, 10, 1064. [CrossRef]

27. Zhang, F.; Zhao, Q.; Zhou, J.; Wang, S. Polarization and incidence insensitive dielectric electromagnetically induced transparency metamaterial. Opt. Express 2013, 21, 19675-19680. [CrossRef]

28. Wang, Q.; Yu, L.; Gao, H.; Chu, S.; Peng, W. Electromagnetically induced transparency in an all-dielectric nano-metamaterial for slow light application. Opt. Express 2019, 27, 35012-35026. [CrossRef]

29. Hu, J.; Lang, T.; Hong, Z.; Shen, C.; Shi, G. Comparison of Electromagnetically Induced Transparency Performance in Metallic and All-Dielectric Metamaterials. J. Light. Technol. 2018, 36, 2083-2093. [CrossRef]

30. Han, B.; Li, X.; Sui, C.; Diao, J.; Jing, X.; Hong, Z. Analog of electromagnetically induced transparency in an E-shaped all-dielectric metasurface based on toroidal dipolar response. Opt. Mater. Express 2018, 8, 2197-2207. [CrossRef]

31. Ma, T.; Huang, Q.; He, H.; Zhao, Y.; Lin, X.; Lu, Y. All-dielectric metamaterial analogue of electromagnetically induced transparency and its sensing application in terahertz range. Opt. Express 2019, 27, 16624-16634. [CrossRef]

32. Zhu, L.; Zhao, X.; Miao, F.J.; Ghosh, B.K.; Dong, L.; Tao, B.R.; Meng, F.Y.; Li, W.N. Dual-band polarization convertor based on electromagnetically induced transparency (EIT) effect in all-dielectric metamaterial. Opt. Express 2019, 27, 12163-12170. [CrossRef]

33. Zhang, Z.; Yang, J.; Bai, W.; Han, Y.; He, X.; Huang, J.; Chen, D.; Xu, S.; Xie, W. All-optical switch and logic gates based on hybrid silicon-Ge2Sb2Te5 metasurfaces. Appl. Opt. 2019, 58, 7392-7396. [CrossRef]

34. Petronijevic, E.; Sibilia, C. All-optical tuning of EIT-like dielectric metasurfaces by means of chalcogenide phase change materials. Opt. Express 2016, 24, 30411-30420. [CrossRef]

35. Wei, Z.; Li, X.; Zhong, N.; Tan, X.; Zhang, X.; Liu, H.; Meng, H.; Liang, R. Analogue Electromagnetically Induced Transparency Based on Low-loss Metamaterial and its Application in Nanosensor and Slow-light Device. Plasmonics 2017, 12, 641-647. [CrossRef]

36. Yang, Y.; Kravchenko, I.I.; Briggs, D.P.; Valentine, J. All-dielectric metasurface analogue of electromagnetically induced transparency. Nat. Commun. 2014, 5, 5753. [CrossRef] [PubMed]

37. Liu, H.; Guo, C.; Vampa, G.; Zhang, J.L.; Sarmiento, T.; Xiao, M.; Bucksbaum, P.H.; Vučković, J.; Fan, S.; Reis, D.A. Enhanced high-harmonic generation from an all-dielectric metasurface. Nat. Phys. 2018, 14, 1006-1010. [CrossRef]

38. Zhang, J.; Liu, W.; Yuan, X.; Qin, S. Electromagnetically induced transparency-like optical responses in all-dielectric metamaterials. J. Opt. 2014, 16, 125102. [CrossRef]

39. Länk, N.O.; Verre, R.; Johansson, P.; Käll, M. Large-Scale Silicon Nanophotonic Metasurfaces with Polarization Independent Near-Perfect Absorption. Nano Lett. 2017, 17, 3054-3060. [CrossRef] [PubMed]

40. Evlyukhin, A.B.; Reinhardt, C.; Chichkov, B. Multipole light scattering by nonspherical nanoparticles in the discrete dipole approximation. Phys. Rev. B 2011, 84, 235429. [CrossRef] 